\title{
Therapeutic effects of simvastatin combined with kallistatin treatment for pediatric burn patients with sepsis
}

\author{
YEFENG DAI and XUFEI ZHAO
}

Department of Burns and Plastic Surgery, Children's Hospital Affiliated to

The Medical College of Zhejiang University, Hangzhou, Zhejiang 310000, P.R. China

Received March 23, 2017; Accepted November 3, 2017

DOI: 10.3892/etm.2018.5791

\begin{abstract}
The aim of the present study was to examine the combined efficacy of simvastatin and kallistatin treatment for pediatric burn sepsis. A total of 72 pediatric patients with burn sepsis were recruited and randomly divided into 3 groups, receiving simvastatin (40 $\mathrm{mg} /$ day), kallistatin $(20 \mathrm{mg} /$ day $)$ or combined treatment. ELISA, reverse transcription-quantitative polymerase chain reaction, western blotting and flow cytometry were used to analyze the therapeutic effects of simvastatin and kallistatin. The results revealed that combined treatment in pediatric burn sepsis patients decreased the inflammatory cytokine tumor necrosis factor $\alpha$ and interleukin (IL) $-1 \beta$ serum levels, whereas it increased IL-10 and human leukocyte antigen-D related levels. In addition, administration of combined simvastatin and kallistatin decreased the blood urea nitrogen and serum creatinine levels in the patients. It was also demonstrated that Toll-like receptor 4 expression on the surface of monocytes was markedly decreased, while suppressor of cytokine signaling-3 expression was increased in the combined treatment group as compared with the kallistatin or simvastatin treatment alone. Combined treatment also promoted human endothelial cell (HEC) growth compared with the single treatment groups and inhibited the high mobility group box-1 (HMGB1) levels, HMGB1-induced nuclear factor- $\kappa \mathrm{B}$ activation and inflammatory gene expression levels in these cells. The study further demonstrated that combined treatment significantly decreased HEC apoptosis through the upregulation of B-cell lymphoma 2 (Bcl-2) and P53 expression levels, as well as downregulation of $\mathrm{Bcl}-2$-associated $\mathrm{X}$ protein and caspase-3 levels. In conclusion, these observations indicated that combined treatment with simvastatin and kallistatin
\end{abstract}

Correspondence to: Dr Xufei Zhao, Department of Burns and Plastic Surgery, Children's Hospital Affiliated to The Medical College of Zhejiang University, 3333 Binsheng Road, Binjiang, Hangzhou, Zhejiang 310000, P.R. China

E-mail: 6506023@zju.edu.cn

Key words: pediatric burn sepsis, simvastatin, kallistatin, human endothelial cells, inflammation, apoptosis inhibited HEC apoptosis, which may be a potential therapeutic strategy for the treatment of pediatric burn sepsis patients.

\section{Introduction}

Sepsis is one of the most common complications that occur in pediatric patients following extensive burns (1). In addition, infection is one of the most common and severe complications of a major burn injury associated with pediatric burn sepsis (2). Clinical data have indicated that sepsis occurs in $>30 \%$ of the pediatric burn patients (3). A previous study reviewed the characteristics of patients with pediatric burn sepsis, including patient categorization, diagnosis and treatment and it was identified that the mortality rate of pediatric patients with burn sepsis is significantly higher mortality than that of adult patients (4). Although an increasing number of strategies have been explored for the treatment of pediatric burn sepsis, the mortality rate remains high $(5,6)$. Additionally, the physiological characteristics, drug treatment and inflammatory responses to burn injury in pediatric patients differ significantly compared with those in adult patients (7-9). Therefore, it is important to develop more effective therapeutic strategies and drugs for the treat pediatric patients with burn sepsis in order to improve their survival rate.

Simvastatin is a statin that is a 3-hydroxy-3-methylglutaryl coenzyme A reductase inhibitor, which is currently used to manage blood cholesterol levels and prevent cardiovascular disease $(10,11)$. It has been reported that simvastatin increases the survival of patients with sepsis or infection by inhibiting sepsis-induced mortality and preventing acute kidney injury (12). It has also been demonstrated that induces anti-inflammatory effects in abdominal sepsis (13). Furthermore, simvastatin treatment improves the survival rate in a murine model of burn sepsis by elevating interleukin (IL)-6 levels (14). However, simvastatin treatment alone is not sufficient to attenuate inflammation and the pathological processes of pediatric burn sepsis patients in clinical practice (15).

Kallistatin is an anthropogenic kallikrein-binding plasma protein that has been demonstrated to exert multiple functions, including inhibition of inflammation, oxidative stress and apoptosis in endothelial cells (16). A previous study revealed that kallistatin presented beneficial anti-inflammation and anti-fibrosis effects by decreasing the antioxidative stress and inhibiting the reactive oxygen species-induced expression 
of proinflammatory cytokines and transforming growth factor- $\beta 1$ (17). In addition, kallistatin treatment inhibited vascular inflammation by antagonizing tumor necrosis factor $\alpha$ ( TNF- $\alpha)$-induced nuclear factor $\kappa \mathrm{B}(\mathrm{NF}-\kappa \mathrm{B})$ activation (18). Furthermore, human kallistatin administration reduced organ injury, as well as inflammatory responses and improves the survival rate in a mouse model of polymicrobial sepsis (19). However, further investigations are required to examine the efficacy of kallistatin in the treatment of pediatric burn sepsis patients.

In the present study, combined treatment with simvastatin and kallistatin was investigated in pediatric burn sepsis therapy. In addition, the study analyzed the anti-inflammatory and anti-apoptotic effects of the combined treatment in human endothelial cells (HECs) collected from the pediatric burn sepsis patients.

\section{Materials and methods}

Study design, subjects and sampling. A total of 72 pediatric patients with burn sepsis from the Children's Hospital Affiliated to the Medical College of Zhejiang University were recruited in the present retrospective study between January 2014 and May 2015. The patients included 30 girls and 42 boys aged 2.8-10.4 years old. The inclusion criteria for the individuals with severe pediatric burn sepsis were diagnosed by inflammatory marks and hemodynamic indexes (20). The inclusion criteria for the current study were patients $<12$ years old with severe pediatric burn sepsis diagnosed using systemic inflammatory response syndrome criteria (21) were included in the present study. Pediatric patients with congenital heart disease, renal failure or cancer were excluded from the study. The study protocol was approved by the Central Ethics Committee of the Children's Hospital Affiliated to the Medical College of Zhejiang University (Hangzhou, China). Written informed consent was obtained from the parents of all included patients. The body temperature of patients was recorded every day and used to calculate the frequency of fever in the current study.

Drug administration. The 72 included patients were randomized into three treatment groups as follows: A simvastatin $(n=30)$, kallistatin $(n=26)$ and combination treatment $(n=16)$ group. Patients in the simvastatin group received $40 \mathrm{mg}$ /day simvastatin orally (Jiangsu Yellow River Pharmaceutical Co. Ltd., Suqian, China), while patients in the kallistatin group received $20 \mathrm{mg} /$ day kallistatin (Wuhan Huamei Biological Engineering Co., Ltd., Wuhan, China). Patients in the combination group received $40 \mathrm{mg} /$ day simvastatin and $20 \mathrm{mg} /$ day kallistatin orally. The treatment lasted 28 days and all the patients completed the study following a 28 day follow-up period.

ELISA. Plasma samples were prepared by centrifugation of peripheral venous blood at $2,000 \times \mathrm{g}$ for $10 \mathrm{~min}$ at $4^{\circ} \mathrm{C}$. Blood was centrifuged immediately following collection from pediatric patients with burn sepsis treated with simvastatin and/or kallistatin on days $0,7,14,21$ and 28. The serum levels of blood urea nitrogen (cat. no., EIABUN, Thermo Fisher Scientific, Inc.), creatinine, (cat. no., EIACUN, Thermo Fisher Scientific, Inc.), TNF- $\alpha$ (cat. no., DTA00C,
Bio-Rad Laboratories, Inc., Hercules, CA, USA), IL-1 $\beta$ (cat. no., DY201, Bio-Rad Laboratories, Inc.), IL-6 (cat. no., D6050, Bio-Rad Laboratories, Inc.), human leukocyte antigen-D related (HLA-DR; cat. no., CSB-EL010497HU, Cusabio, Wuhan, China) and procalcitonin (PCT; cat. no., EHPCT; Thermo Fisher Scientific, Inc.) were then analyzed using the corresponding ELISA kits according to the manufacturer's protocol. The serum concentration levels of IL-1 $\beta$, TNF- $\alpha$, IL-6, HLA-DR and PCT were measured using a microplate reader at $570 \mathrm{~nm}$.

Inflammation severity score. On days 0, 7, 14, 21 and 28, treatment efficacy was evaluated according to the inflammation severity score as the efficacy criteria. The mean inflammation severity score of patients was evaluated according to previous studies $(22,23)$.

Revised trauma score (RTS). The RTS scoring system examines the injury severity of burn sepsis patients in three groups and includes evaluation of the Glasgow Coma scale (24), systolic blood pressure and respiratory rate, evaluated on days 0 and 14. The score ranges between 0 and 12. In the 'simple triage and rapid treatment' triage, a patient with an RTS score of 12 is considered to require delayed care, a score of 11 indicates that urgent care is required and a score of 3-10 indicates that immediate care is required. Patients with an RTS of $<3$ should receive emergency care, which includes the administration of anti-inflammatory medication and antibiotics and admittance into the intensive care unit, as survival is highly unlikely without a significant amount of medication.

HEC culture. HECs were harvested from the pediatric burn sepsis patients ( $\mathrm{n}=3$ in each group) as previously described (25). Briefly, on day 30, endothelial specimens were minced with dissecting scissors into $\leq 2-\mathrm{mm}$ sections followed by digestion with collagenase type IV $(2 \mathrm{mg} / \mathrm{ml}$; Worthington Biochemical Corp., Lakewood, NJ, USA), Dispase II (1.2 U/ml; Worthington Biochemical Corp.) and $2 \mathrm{mM} \mathrm{CaCl}_{2}$ in phosphate-buffered saline (PBS) at $37^{\circ} \mathrm{C}$ for $60 \mathrm{~min}$. Enzymes and thus, the reaction was terminated following the addition of endothelial basal medium (EBM; Thermo Fisher Scientific, Inc., Waltham, MA, USA) with $10 \%$ fetal bovine serum (FBS; Gibco; Thermo Fisher Scientific, Inc.). Next, cells were filtered through a sterile $50-\mu \mathrm{m}$ nylon mesh cell strainer (Thermo Fisher Scientific, Inc.), centrifuged at $300 \mathrm{x}$ g for $5 \mathrm{~min}$ at $4^{\circ} \mathrm{C}$ and cultured in hemolytic buffer (containing $155 \mathrm{mM} \mathrm{NH}_{4} \mathrm{Cl}$, $10 \mathrm{mM} \mathrm{KHCO}_{3}$ and $0.1 \mathrm{mM}$ EDTA in $\mathrm{H}_{2} \mathrm{O}$ ) for $15 \mathrm{~min}$ at $37^{\circ} \mathrm{C}$. Subsequently, the cells were cultured in complete EBM with 20\% FBS, growth factor supplement kit (Lonza Group Ltd., Basel Switzerland) and penicillin $100 \mathrm{U} / \mathrm{ml}$ (Gibco; Thermo Fisher Scientific, Inc.) for $12 \mathrm{~h}$ at $37^{\circ} \mathrm{C}$.

MTT assay. HECs $\left(5 \times 10^{3}\right.$ cells/well) isolated from pediatric patients with burn sepsis subsequent to simvastatin and/or kallistatin treatment were grown in 12-well plates to $295 \%$ monolayer cells for $24 \mathrm{~h}$ at $37^{\circ} \mathrm{C}$. MTT at a concentration of $5 \mathrm{mg} / \mathrm{ml}$ (50 $\mu \mathrm{l}$; Amresco, LLC, Solon, OH, USA), was added to the cells and incubated for $4 \mathrm{~h}$. DMSO $(100 \mu \mathrm{l})$ was then added and incubated for $30 \mathrm{~min}$ to dissolve the precipitate following removal of the supernatant. The results were determined using 
a spectrophotometer (Bio-Rad Laboratories, Inc., Hercules, CA, USA) at $570 \mathrm{~nm}$.

Reverse transcription-quantitative polymerase chain reaction $(R T-q P C R)$ analysis. Total RNA was obtained from HECs using the RNAeasy Mini kit (Qiagen, Hilden, Germany). RNA was reversed transcribed using a PrimeScript RT Master Mix kit (Takara Bio, Inc., Otsu, Japan). All the forward and reverse primers were synthesized by Thermo Fisher Scientific, Inc., and were as follows: TLR4, 5'-AAACTCAGCAAAGTCCCT GATGAC-3' (forward) and 5'-CGTAGAAACTGTAAGTCG TTGACAG-3' (reverse); SOCS3, 5'-ACCTTCAGCTCCAAA AGCGAGTAC-3' (forward) and 5'-CGCTCCAGTAGAATC CGCTCTC-3' (reverse); high mobility group box-1 (HMGB1), 5'-GGCGAGCATCCTGGCTTATC-3' (forward) and 5'-AGG CAGCAATATCCTTCTCATAC-3' (reverse); $\beta$-actin, 5'-AGG CCGGTGCTGAGTATGTC-3' (forward) and 5'-TGCCTG CTTCACCACCTTCT-3' (reverse). qPCR was performed using a qPCR system (Invitrogen; Thermo Fisher Scientific, Inc.) with SYBR Green Master Mix (Invitrogen; Thermo Fisher Scientific, Inc.) according to the manufacturer's protocol. A total of 45 amplification cycles were performed, including $94^{\circ} \mathrm{C}$ for $30 \mathrm{sec}$, denaturation at $95^{\circ} \mathrm{C}$ for $5 \mathrm{sec}$, primer annealing at $63^{\circ} \mathrm{C}$ for $5 \mathrm{sec}$ with touchdown to $54^{\circ} \mathrm{C}$ for $15 \mathrm{sec}$ and applicant extension at $72^{\circ} \mathrm{C}$ for $10 \mathrm{sec}$. The relative mRNA expression changes were calculated by the $2^{-\Delta \Delta C q}$ method (26) and the results are expressed as the fold of $\beta$-actin, which served as the internal control.

Western blotting. HECs were homogenized in lysate buffer containing protease-inhibitor (Sigma-Aldrich; Merck KGaA) and then centrifuged at $6,000 \mathrm{x}$ g at $4^{\circ} \mathrm{C}$ for $10 \mathrm{~min}$. The supernatant was collected and used for the analysis of the target protein expression. Protein concentration was measured using a BCA protein assay kit (Thermo Fisher Scientific Inc.). Protein samples $(10 \mu \mathrm{g})$ were separated on $12.5 \%$ SDS-PAGE and transferred onto polyvinylidene difluoride membranes (Millipore, Massachusetts, USA). Membrane were blocked with $5 \%$ skimmed milk for $1 \mathrm{~h}$ at $37^{\circ} \mathrm{C}$ and then incubated with the following rabbit anti-human primary antibodies: B-cell lymphoma 2 (Bcl-2; 1:500; cat. no., ab692), P53 (1:500; cat. no., ab26), Bcl-2-associated X protein (1:500; cat. no., ab22048), caspase-3 (1:500, cat. no., ab13847) and $\beta$-actin (1:500; cat. no., ab8227; all purchased from Abcam, Shanghai, China) for $12 \mathrm{~h}$ at $4^{\circ} \mathrm{C}$. Samples were then incubated with horseradish peroxidase (HRP)-conjugated anti-rabbit IgG antibody (1:5,000, PV-6001, ZSGB-BIO, Beijing, China) for $24 \mathrm{~h}$ at $4^{\circ} \mathrm{C}$. The membranes were then visualized using WesternBright ECL Chemiluminescent HRP Substrate (Advansta, Menlo Park, CA, USA). The density of the bands was analyzed using Quantity one software version 4.62 (Bio-Rad Laboratories, Inc.).

Analysis of TLR4-positive cells. Blood samples were collected on days $0,7,14,21$ and 28 following simvastatin and/or kallistatin treatments. Blood samples were placed into a test tube containing EDTA and peripheral blood mononuclear cells were isolated by density-gradient centrifugation using Ficoll-Paque Premium (GE Healthcare Life Sciences) according to the manufacturer's protocol. Samples were blocked by $5 \%$ bovine serum albumin (Thermo Fisher Scientific, Inc.) for $2 \mathrm{~h}$ at $4^{\circ} \mathrm{C}$ were then incubated with anti-TLR4 (cat. no. 551964, 1:1,000) and anti-CD14 antibodies (cat. no. 555397; 1:1,000, BD Biosciences, Franklin Lakes, NJ, USA) for $1 \mathrm{~h}$ at $4^{\circ} \mathrm{C}$ and then with anti-immunoglobulin G H\&L secondary antibody (Alexa Fluor ${ }^{\circledR} 488$; cat. no., ab150117, Abcam) for $2 \mathrm{~h}$ at $4^{\circ} \mathrm{C}$ following washing with PBS three times at room temperature. TLR4/CD14 double-positive peripheral blood mononuclear cells were then identified using a flow cytometer. The number of TLR4-positive peripheral blood mononuclear cells was calculated in terms of the mean fluorescent intensity.

Apoptosis assay. HECs were isolated from pediatric patients with burn sepsis on days $0,7,14,21$ and 28 after simvastatin and/or kallistatin treatment. HECs were adjusted to $1 \times 10^{6}$ cells/ml with PBS and labeled with Annexin V-FITC and PI using a kit (BD Biosciences) according to the manufacturer's protocol. Subsequently, cells were analyzed with a FACScan flow cytometer (BD Biosciences). The treatments were performed in triplicate and the percentage of labeled cells undergoing apoptosis in each group was determined and calculated. The ratios of apoptotic cells were analyzed using Expo32-ADC v. 1.2B software (Beckman Coulter, Inc., Brea, CA, USA).

$N F-\kappa B$ activation. HECs were isolated from pediatric patients with burn sepsis on days 0 and 28 following simvastatin and/or kallistatin treatment. Subsequently, HECs were seeded at a density of $2 \times 10^{5}$ cells/well in 24-well plates and cultured in EBM. The 3'-untranslated region (3'-UTR) sequence of $\mathrm{NF}-\mathrm{\kappa B}$, predicted to the effects of simvastatin and kallistatin on NF- $\mathrm{kB}$ activity, was cloned into the pGL3 control vector (Promega Corporation, Madison, WI, USA). These constructs were designated as wild-type (wt) NF-kB-3'-UTR or mutant NF- $\kappa B-3 '-U T R$, respectively. For the reporter assay, HECs $\left(1 \times 10^{4}\right)$ were seeded in 24-well plates and transfected with the aforementioned constructs or negative control (mutant NF-кB-3'-UTR) using lipofectamine 2000 (Invitrogen; Thermo Fisher Scientific, Inc.). After 48 h, the cells were collected and Renilla luciferase activity in cells was measured using the Dual-Luciferase Reporter assay system (Promega Corporation) following the manufacturer's protocol, as described previously (27). Luciferase activity was normalized to that of Renilla luciferase. Results were obtained from three independent experiments performed in duplicate.

Statistical analysis. Continuous variables are expressed as the mean \pm standard deviation and were analyzed by Student's t-test. All data were analyzed using SPSS 19.0 statistical software (IBM Corp., Armonk, NY, USA) along with the use of Microsoft Excel (Microsoft Corp., Redmond, WA, USA). Unpaired data were determined by Student's t-test, while comparisons of data between multiple groups were conducted by analysis of variance. A P-value of $\leq 0.05$ was considered to indicate a difference that was statistically significant.

\section{Results}

Effect of combined treatment with simvastatin and kallistatin on the levels of inflammation-associated cytokines in pediatric burn sepsis patients. Pediatric burn sepsis patients received simvastatin, kallistatin or combined treatment with 

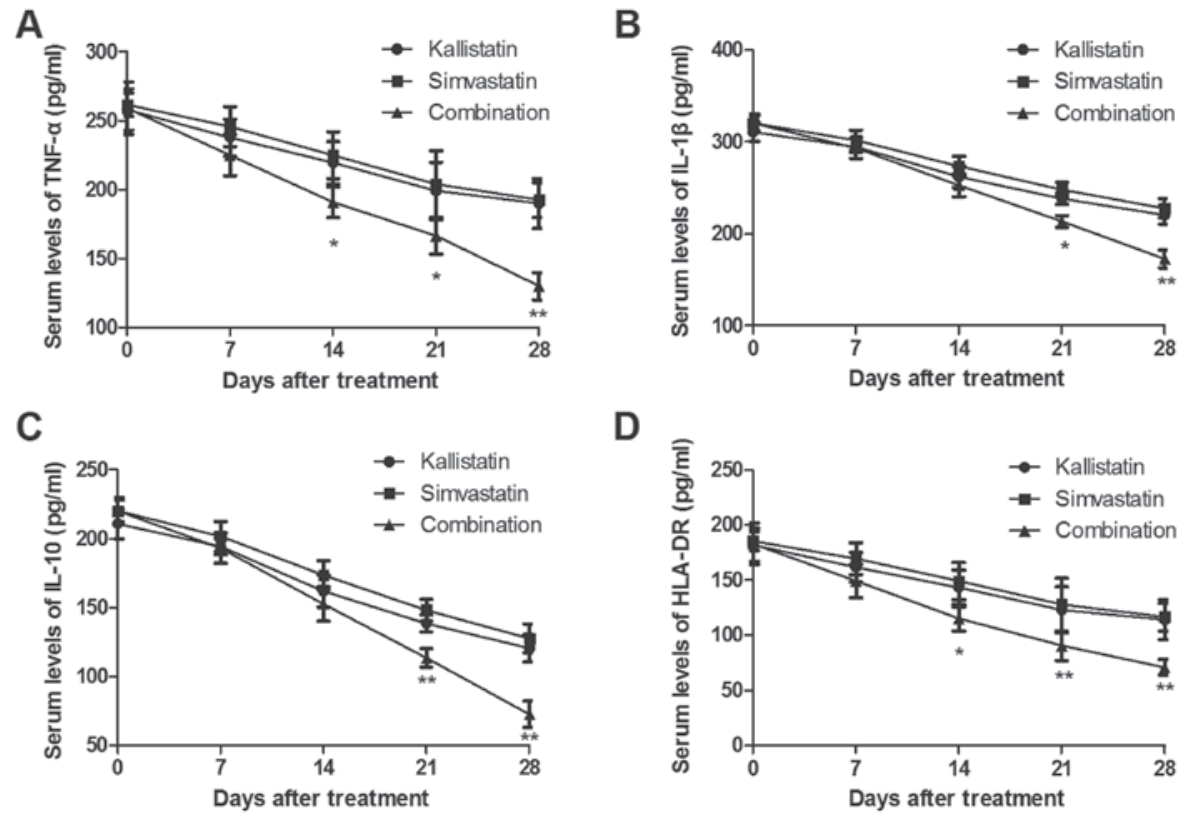

Figure 1. Combined treatment with simvastatin and kallistatin improves inflammatory cytokine levels in pediatric burn sepsis patients. Combined treatment decreased the serum levels of (A) TNF- $\alpha$ and (B) IL-1 $\beta$, while it increased the levels of anti-inflammatory cytokines (C) IL-10 and (D) HLA-DR in the pediatric patients with burn sepsis. ${ }^{*} \mathrm{P}<0.05$ and ${ }^{* *} \mathrm{P}<0.01$ vs. single treatment groups. TNF- $\alpha$, tumor necrosis factor $\alpha$; IL, interleukin; HLA-DR, human leukocyte antigen-D related.

simvastatin and kallistatin and the levels of inflammatory cytokines TNF- $\alpha$, IL-1 $\beta$, IL-10 and HLA-DR in the serum were determined on days $0,7,14,21$ and 28. As presented in Fig. 1A and $\mathrm{B}$, the serum levels of TNF- $\alpha$ and IL-1 $\beta$ were significantly decreased in the combined treatment group, compared with those that received simvastatin or kallistatin alone. However, the anti-inflammatory cytokines IL-10 and HLA-DR were markedly downregulated in the serum of pediatric burn sepsis patients subsequent to combined treatment (Fig. 1C and D). Furthermore, the results indicated that the levels of TNF- $\alpha$ and IL-1 $\beta$ were not significantly altered between the simvastatin alone and kallistatin alone group.

Effect of combined treatment with simvastatin and kallistatin on blood urea nitrogen and serum creatinine levels, PCT and RTS in pediatric burn sepsis patients. Several important biochemical indicators were investigated in pediatric patients with burn sepsis subsequent to receiving simvastatin, kallistatin or combined treatment. The results revealed that combined treatment significantly decreased blood urea nitrogen in pediatric burn sepsis patients on days 21 and 28 and exhibited decreased serum creatinine levels on days 14, 21 and 28 (Fig. 2A and B). Furthermore, the results indicated that the PCT level was evidently downregulated by combined treatment with simvastatin and kallistatin in the serum of burn sepsis patients from day 14 (Fig. 2C). The RTS values were also significantly improved on days 14, 21 and 28 in patients receiving combined treatment (Fig. 2D). These outcomes suggest that combined treatment with simvastatin and kallistatin improves the prognosis of pediatric burn sepsis patients.

Efficacy of combined treatment with simvastatin and kallistatin on the improvement of inflammation-associated gene levels in pediatric burn sepsis patients. The present study further analyzed alterations in the expression levels of inflammation-associated genes in HECs. The results revealed that combined treatment with simvastatin and kallistatin significantly promoted the viability of HECs compared with that of the single treatment groups (Fig. 3A). Treatment with simvastatin and kallistatin increased the viability of HECs compared with the groups treated with simvastatin or kallistatin alone. In addition, it was demonstrated that combined treatment with simvastatin and kallistatin improved the morphology of HECs (Fig. 3B). The results further indicated that TLR4 expression on the surface of monocytes was markedly decreased and SOCS3 expression was markedly increased in the combined treatment group compared with the levels in the kallistatin or simvastatin only groups on days 14, 21 and 28 (Fig. 3C and D). Notably, it was demonstrated that HMGB1 levels and HMGB1-induced $\mathrm{NF}-\kappa \mathrm{B}$ p65 activation were inhibited in HECs in three groups (Fig. 3E and F), with a significant difference observed in the combination treatment as compared with the kallistatin or simvastatin groups after 14-day treatment. Furthermore, it was observed that the inflammation severity score was significantly decreased following combined treatment as compared with that in the single treatment groups (Fig. 3G). No significant differences were observed between the kallistatin and simvastatin groups. These outcomes suggested that combined treatment with simvastatin and kallistatin improved the levels inflammation-associated genes and inflammation severity score in HECs in pediatric burn sepsis patients.

Anti-apoptotic efficacy of combined treatment with simvastatin and kallistatin in pediatric burn sepsis patients. The anti-apoptotic efficacy of combined treatment with simvastatin and kallistatin was investigated in HECs obtained from pediatric burn sepsis patients. As presented in Fig. 4A, it was observed that combined treatment with kallistatin and 

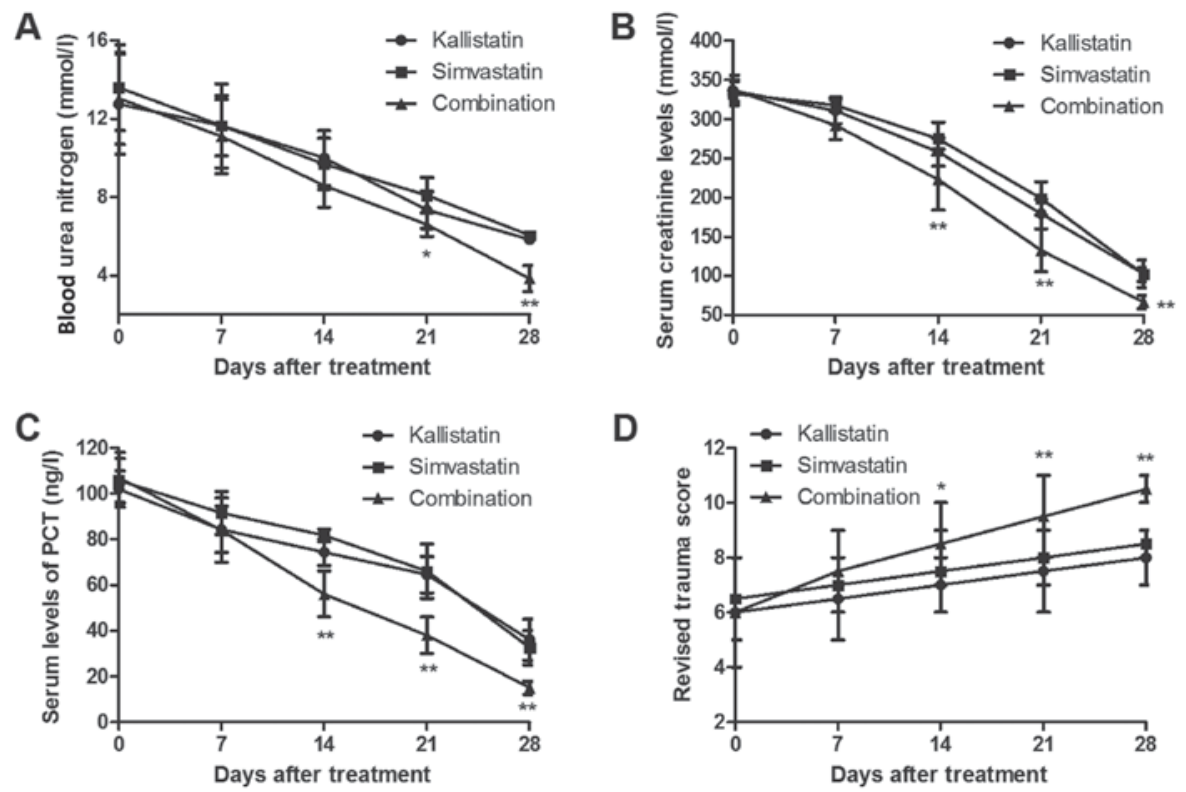

Figure 2. Combined treatment with simvastatin and kallistatin improves blood urea nitrogen and serum creatinine levels in pediatric burn sepsis patients. (A) Blood urea nitrogen and (B) serum creatinine levels were decreased by combined treatment in pediatric burn sepsis patients during hospitalization. (C) Serum PCT was also downregulated by the combined treatment. (D) The Revised Trauma Score was increased following combined treatment with simvastatin and kallistatin in pediatric burn sepsis patients during hospitalization. ${ }^{*} \mathrm{P}<0.05$ and ${ }^{* *} \mathrm{P}<0.01$ vs. single treatment groups. PCT, procalcitonin.

simvastatin significantly decreased the apoptosis of HECs on day 28 compared with either treatment alone. Additionally, combined treatment upregulated the Bcl-2 and P53 expression in HECs on day 28 (Fig. 4B), while the expression of the pro-apoptotic Bax and caspase-3 proteins were downregulated (Fig. 4C). Notably, the findings indicated that combined treatment significantly improved the fever frequency on day 28 compared with the single treatment groups (Fig. 4D). These results suggested that combined treatment with simvastatin and kallistatin inhibited the apoptosis of HECs and improved the fever frequency in pediatric burn sepsis patients.

\section{Discussion}

Burn sepsis is a systemic inflammatory response syndrome and pediatric patients with burn wound-induced sepsis have a relative higher mortality rate compared with that of adult patients with sepsis (2). Currently, an increasing number of drugs are applied for the treatment of pediatric burn sepsis $(28,29)$. Previous studies have indicated that simvastatin is able to attenuate the sepsis-induced blood-brain barrier integrity loss, which also inhibits the apoptosis of endothelial cells induced by sepsis by upregulating the expression of Bcl-2 and downregulating Bax $(15,30)$. In addition, a previous study suggested that kallistatin is able to protect HECs against inflammation and apoptosis, as well as improve sepsis-associated acute lung injury (31). In the current study, in order to improve the therapeutic efficacy of clinical treatment, combined treatment with simvastatin and kallistatin, or treatment with simvastatin or kallistatin alone were administered to patients with burn sepsis. The results of the current study indicated that combined treatment with simvastatin and kallistatin may be a potential therapeutic regimen for the treatment of pediatric burn sepsis patients in clinical practice.
Inflammatory response is a major cause of mortality in pediatric patients with burn sepsis (32). The results of the present study revealed that simvastatin treatment decreased TNF- $\alpha$ and IL- $1 \beta$ levels, while it increased the serum levels of the anti-inflammatory cytokines IL-10 and HLA-DR in pediatric burn sepsis patients. A previous study reviewed the inflammatory responses and anti-inflammatory strategies in experimental models of sepsis and hemorrhage, and indicated that the regulation of inflammatory pathways contributed to the treatment of pediatric burn sepsis through different pathophysiological processes (33). Simvastatin treatment has a beneficial effect in patients with sepsis and severe sepsis by decreasing the inflammation (34). In HECs isolated from pediatric burn sepsis patients, simvastatin treatment resulted in markedly decreased TLR4, HMGB1 and NF-kB levels, as well as in increased SOCS3 expression.

It has been reported that kallistatin may be a potential therapeutic agent to treat pediatric burn sepsis (31). In the present study, the therapeutic effects of kallistatin we also investigated in pediatric burn sepsis patients. The outcomes indicated that kallistatin treatment exerted anti-inflammatory effects and inhibited the apoptosis of HECs isolated from pediatric burn sepsis patients, with no marked differences detected between the simvastatin and kallistatin-treated groups. Zhou et al (35) have suggested that kallistatin treatment significantly improved the oxidative stress and inflammation, indicating that kallistatin was able to regulate the inflammation. The current study revealed that kallistatin treatment also decreased the levels of TLR4, HMGB1 and NF- $\mathrm{kB}$, as well as increased SOCS3 expression, in HECs isolated from pediatric burn sepsis patients.

In the present study, it was reported that combined treatment with kallistatin and simvastatin significantly decreased the apoptosis of HECs on day 28. Lee et al (36) have demonstrated that simvastatin presented an anti-apoptotic 
A

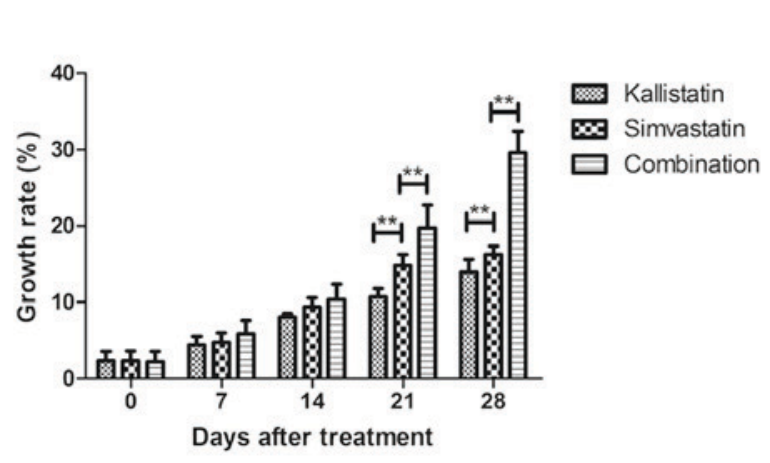

C

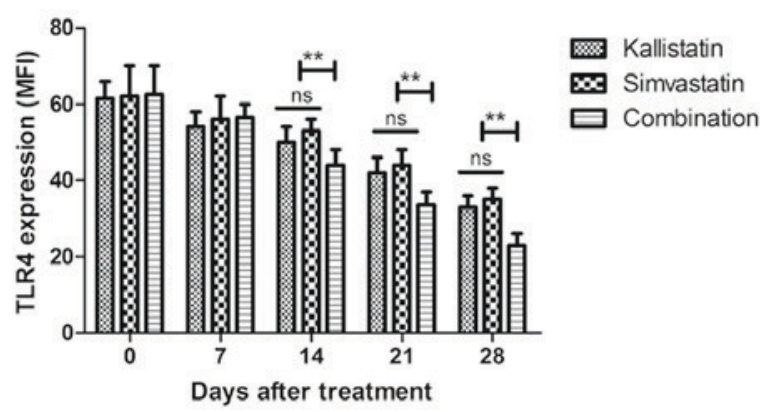

E

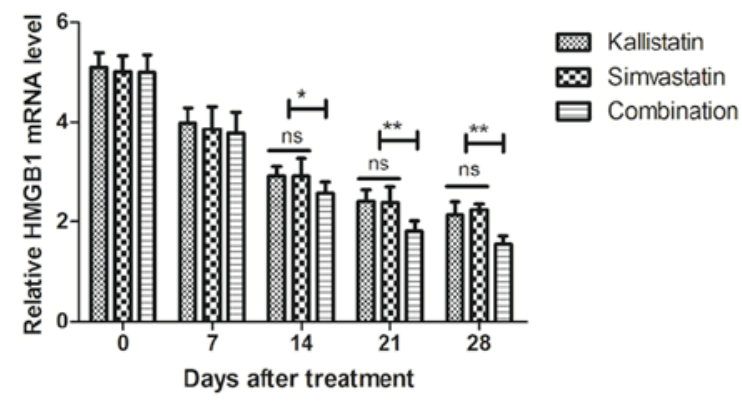

G

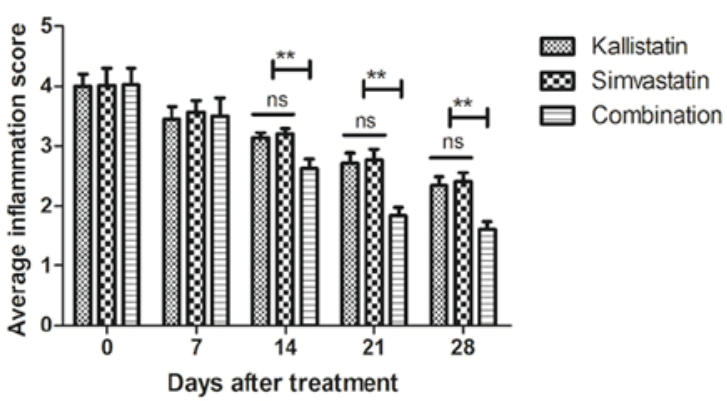

B

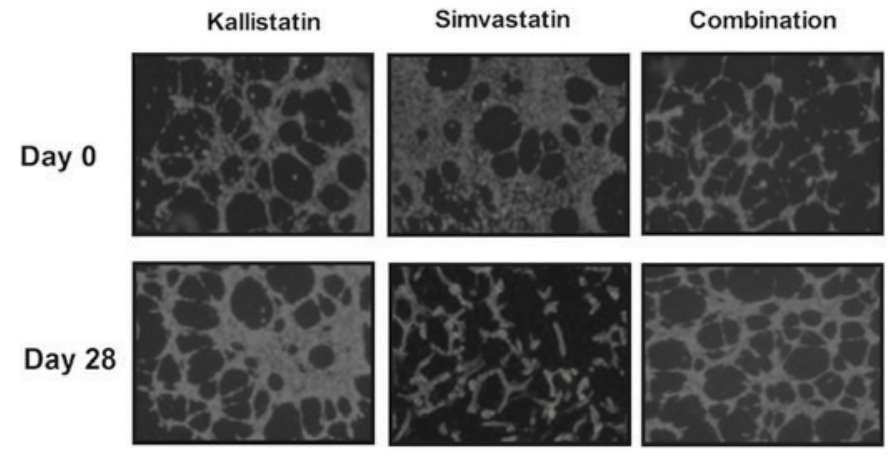

D

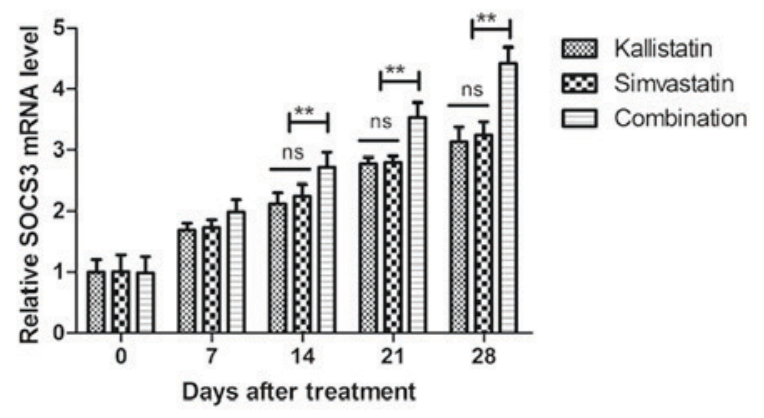

F

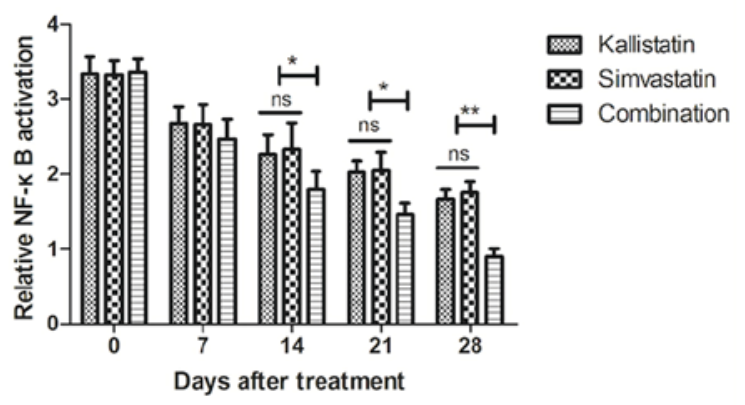

Figure 3. Combined treatment with simvastatin and kallistatin regulates inflammation-associated genes in pediatric burn sepsis patients. (A) The growth rate of HECs was stimulated and (B) their morphology was improved following combined treatment. (C) TLR4 expression on the surface of monocytes was decreased, while (D) SOCS3 serum expression during hospitalization was increased by the combined treatment strategy. (E) HMGB1 mRNA levels were decreased in HECs following simvastatin and/or kallistatin treatment. (F) NF- $\mathrm{kB}$ activation in HECs was increased by the combined treatment determined by dual-luciferase assay. (G) Inflammation was significantly improved subsequent to combined treatment in pediatric burn sepsis patients during hospitalization. ${ }^{*} \mathrm{P}<0.05$ and ${ }^{* *} \mathrm{P}<0.01$ vs. single treatment groups. HECs, human endothelial cells; TLR4, Toll-like receptor 4; SOCS3, suppressor of cytokine signaling-3; HMGB1, high mobility group box-1; NF, nuclear factor; ns, not significant. 
A

A
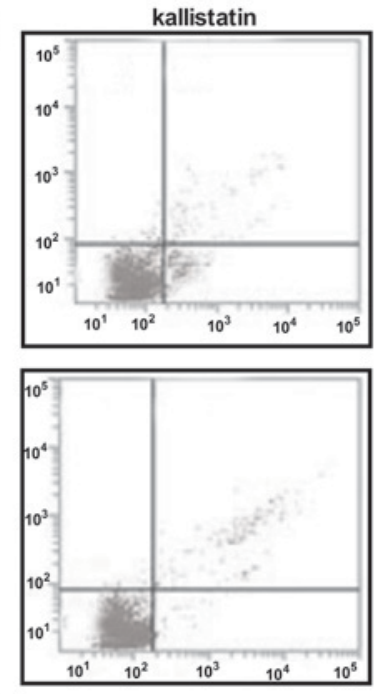
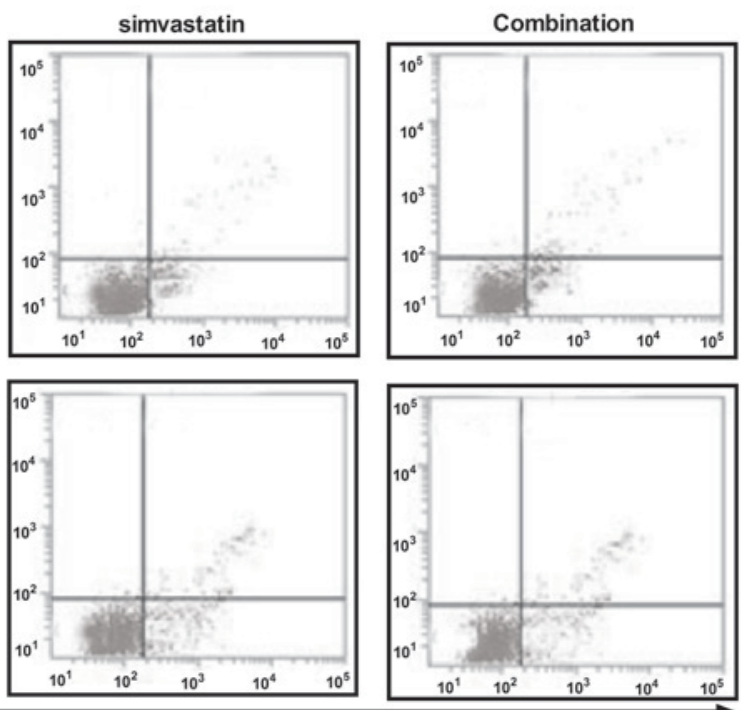

Annexin V-FITC

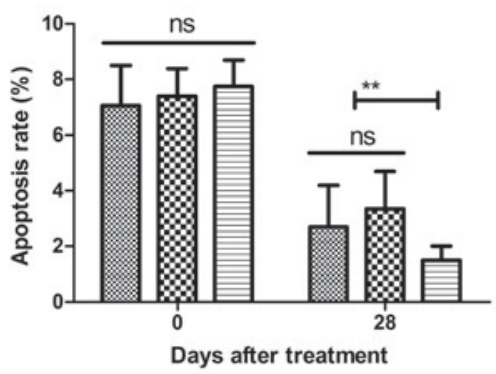

Kallistatin

$\infty$ Simvastatin

$\square$ Combination

Days after treatment

B

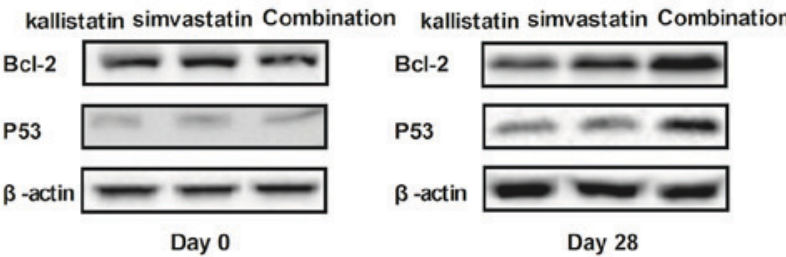

C

D
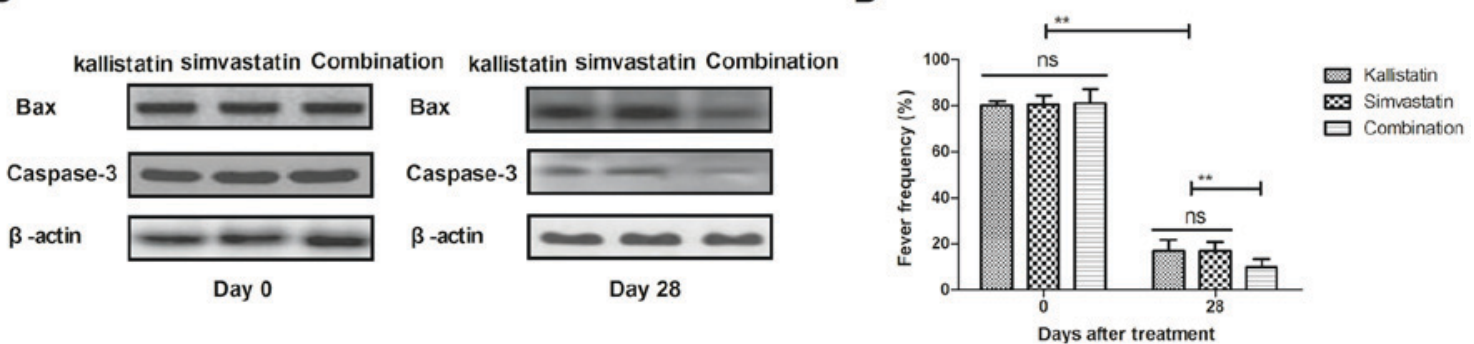

Figure 4. Combined treatment with simvastatin and kallistatin inhibits the apoptosis of HECs in pediatric burn sepsis patients. (A) Flow cytometry results indicated decreased apoptosis of HECs on day 28 after combined treatment. (B) Bcl-2 and P53 expression levels were upregulated, while (C) pro-apoptotic Bax and caspase-3 protein expression levels were downregulated in HECs following combined treatment with kallistatin and simvastatin. (D) Fever frequency was improved in patients receiving with combined treatment compared with that in the single treatment group. ${ }^{* *} \mathrm{P}<0.01$ vs. single treatment groups. HECs, human endothelial cells; Bcl-2, B-cell lymphoma 2; Bax, Bcl-2-associated X protein; ns, not significant.

effect by increasing P53 expression. Another study indicated that simvastatin regulated neuronal apoptosis through the modulation of $\mathrm{Bcl}-2$ expression in a rat quinolinic acid model of Huntington's disease (37). Furthermore, Chao et al (38) indicated that kallistatin protected against myocardial ischemia-reperfusion injury by preventing apoptosis and inflammation. The present study results revealed that combined treatment with kallistatin and simvastatin significantly increased Bcl-2 and P53 expression levels, whereas it decreased Bax and caspase-3 levels in HECs. It was also 
observed that combined treatment significantly improved the incidence of fever as compared with that in the single treatment group.

In conclusion, the present study reported the efficacy of combined treatment with simvastatin and kallistatin in pediatric burn sepsis patients. The outcomes demonstrated that combined treatment exhibited increased beneficial effects for these patients as compared with simvastatin or kallistatin treatment alone in terms of anti-inflammation and anti-apoptosis. These results indicated that combined therapy with simvastatin and kallistatin resulted in successful outcomes for numerous pediatric patients with burn sepsis.

\section{References}

1. Housinger TA, Brinkerhoff $\mathrm{C}$ and Warden GD: The relationship between platelet count, sepsis, and survival in pediatric burn patients. Arch Surg 128: 65-67, 1993.

2. Yu T, Stockmann C, Healy DP, Olson J, Wead S, Neely AN, Kagan RJ, Spigarelli MG and Sherwin CM: Determination of optimal amikacin dosing regimens for pediatric patients with burn wound sepsis. J Burn Care Res 36: e244-e252, 2015 .

3. Sheridan RL: Sepsis in pediatric burn patients. Pediatr Crit Care Med 6 (3 Suppl): S112-S119, 2005.

4. Peng YZ: Clinical characteristics and diagnosis of sepsis in pediatric burn patients. Zhonghua Shao Shang Za Zhi 29: 1-3, 2013 (In Chinese)

5. Scott HF, Paul R and Balamuth F: The spectrum of pediatric sepsis: 'Septicemia' misses severe cases. Ann Emerg Med 66 : 685-686, 2015.

6. Ruth A, McCracken CE, Fortenberry JD and Hebbar KB: Extracorporeal therapies in pediatric severe sepsis: Findings from the pediatric health-care information system. Crit Care 19: 397,2015 .

7. Odetola FO, Gebremariam A and Freed GL: Patient and hospital correlates of clinical outcomes and resource utilization in severe pediatric sepsis. Pediatrics 119: 487-494, 2007.

8. Alexandre-Treilles M, Chenaud M, Kacet N, Ego A and Truffert P; OMBREL de Soins Périnatals de Lille Métropole: Pediatric management of early-onset neonatal sepsis: Guidelines adherence in Lille's perinatal care network. Arch Pediatr 13: 341-345, 2006 (In French).

9. Moloney-Harmon PA: Pediatric sepsis: The infection unto death. Crit Care Nurs Clin North Am 17: 417-429, xi, 2005.

10. Ramadan WH and Kabbara WK: Sitagliptin/Simvastatin: A first combination tablet to treat type 2 diabetes and hypercholesterolemia-a review of its characteristics. Vasc Health Risk Manag 11: $125-132,2015$

11. Taveira-DaSilva AM, Jones AM, Julien-Williams PA, Stylianou M and Moss J: Retrospective review of combined sirolimus and simvastatin therapy in lymphangioleiomyomatosis. Chest 147: 180-187, 2015.

12. Yasuda H, Yuen PS, Hu X, Zhou H and Star RA: Simvastatin improves sepsis-induced mortality and acute kidney injury via renal vascular effects. Kidney Int 69: 1535-1542, 2006.

13. Souza Neto JL, Araújo Filho I, Rego AC, Dominici VA, Azevedo IM, Egito ES, Brandão-Neto J and Medeiros AC: Effects of simvastatin in abdominal sepsis in rats. Acta Cir Bras 21 (Suppl 4): S8-S12, 2006.

14. Beffa DC, Fischman AJ, Fagan SP, Hamrahi VF, Paul KW, Kaneki M, Yu YM, Tompkins RG and Carter EA: Simvastatin treatment improves survival in a murine model of burn sepsis: Role of interleukin 6. Burns 37: 222-226, 2011.

15. Fu H, Wang QS, Luo Q, Tan S, Su H, Tang SL, Zhao ZL and Huang LP: Simvastatin inhibits apoptosis of endothelial cells induced by sepsis through upregulating the expression of Bcl-2 and downregulating Bax. World J Emerg Med 5: 291-297, 2014.

16. Wang CR, Chen SY, Wu CL, Liu MF, Jin YT, Chao L and Chao J: Prophylactic adenovirus-mediated human kallistatin gene therapy suppresses rat arthritis by inhibiting angiogenesis and inflammation. Arthritis Rheum 52: 1319-1324, 2005.
17. Shen B, Hagiwara M, Yao YY, Chao L and Chao J: Salutary effect of kallistatin in salt-induced renal injury, inflammation, and fibrosis via antioxidative stress. Hypertension 51: 1358-1365, 2008.

18. Yin H, Gao L, Shen B, Chao L and Chao J: Kallistatin inhibits vascular inflammation by antagonizing tumor necrosis factor-alpha-induced nuclear factor kappaB activation. Hypertension 56: 260-267, 2010.

19. Li P, Guo Y, Bledsoe G, Yang ZR, Fan H, Chao L and Chao J: Kallistatin treatment attenuates lethality and organ injury in mouse models of established sepsis. Crit Care 19: 200, 2015.

20. Chai JK: Diagnosis and comprehensive management of sepsis after burn. Zhonghua Shao Shang Za Zhi 29: 105-108, 2013.

21. Maguire PJ, Power KA, Downey AF, O'Higgins AC, Sheehan SR and Turner MJ: Evaluation of the systemic inflammatory response syndrome criteria for the diagnosis of sepsis due to maternal bacteremia. Int J Gynaecol Obstet 133: 116-119, 2016.

22. Hautamäki A, Luoma A and Immonen I: Anterior chamber flare during bevacizumab treatment in eyes with exudative age-related macular degeneration. Retina 36: 2183-2190, 2016.

23. Nierhaus A, Linssen J, Wichmann D, Braune S and Kluge S: Use of a weighted, automated analysis of the differential blood count to differentiate sepsis from non-infectious systemic inflammation: The intensive care infection score (ICIS). Inflamm Allergy Drug Targets 11: 109-115, 2012.

24. Kondo Y, Abe T, Kohshi K, Tokuda Y, Cook EF and Kukita I: Revised trauma scoring system to predict in-hospital mortality in the emergency department: Glasgow coma scale, age, and systolic blood pressure score. Crit Care 15: R191, 2011.

25. Hewett PW: Vascular endothelial cells from human micro- and macrovessels: Isolation, characterisation and culture. Methods Mol Biol 467: 95-111, 2009.

26. Livak KJ and Schmittgen TD: Analysis of relative gene expression data using real-time quantitative PCR and the 2(-Delta Delta C(T)) method. Methods 25: 402-408, 2001.

27. Collins PE, O'Carroll C and Carmody RJ: Measurement of $N F-\kappa B$ transcriptional activity and identification of $N F-\kappa B$ cis-regulatory elements using luciferase assays. Methods Mol Biol 1280: 25-43, 2015.

28. Riedel S and Carroll KC: Early identification and treatment of pathogens in sepsis: Molecular diagnostics and antibiotic choice. Clin Chest Med 37: 191-207, 2016.

29. Brown KA, Brown GA, Lewis SM, Beale R and Treacher DF: Targeting cytokines as a treatment for patients with sepsis: A lost cause or a strategy still worthy of pursuit? Int Immunopharmacol 36: 291-299, 2016.

30. Yang CH, Kao MC, Shih PC, Li KY, Tsai PS and Huang CJ: Simvastatin attenuates sepsis-induced blood-brain barrier integrity loss. J Surg Res 194: 591-598, 2015.

31. Lin WC, Chen CW, Huang YW, Chao L, Chao J, Lin YS and Lin CF: Kallistatin protects against sepsis-related acute lung injury via inhibiting inflammation and apoptosis. Sci Rep 5: 12463, 2015.

32. Bird L: Inflammation: Hope for sepsis treatment. Nat Rev Drug Discov 9: 516-517, 2010

33. Cai B, Deitch EA and Ulloa L: Novel insights for systemic inflammation in sepsis and hemorrhage. Mediators Inflamm 2010: 642462, 2010.

34. Shao H, Wang C, Zhu W, Huang X, Guo Z, Zhang H and Qin B: Influence of simvastatin treatment on Toll-like receptor 4 in monocytes of peripheral blood in patients with sepsis and severe sepsis. Zhonghua Wei Zhong Bing Ji Jiu Yi Xue 28: 159-163, 2016 (In Chinese).

35. Zhou S, Sun Y, Zhuang Y, Zhao W, Chen Y, Jiang B, Guo C, Zhang Z, Peng $\mathrm{H}$ and Chen Y: Effects of kallistatin on oxidative stress and inflammation on renal ischemia-reperfusion injury in mice. Curr Vasc Pharmacol 13: 265-273, 2015.

36. Lee SK, Kim YC, Song SB and Kim YS: Stabilization and translocation of p53 to mitochondria is linked to Bax translocation to mitochondria in simvastatin-induced apoptosis. Biochem Biophys Res Commun 391: 1592-1597, 2010.

37. Patassini S, Giampà C, Martorana $\mathrm{A}$, Bernardi $\mathrm{G}$ and Fusco FR: Effects of simvastatin on neuroprotection and modulation of Bcl-2 and BAX in the rat quinolinic acid model of Huntington's disease. Neurosci Lett 448: 166-169, 2008.

38. Chao J, Yin H, Yao YY, Shen B, Smith RS Jr and Chao L: Novel role of kallistatin in protection against myocardial ischemia-reperfusion injury by preventing apoptosis and inflammation. Hum Gene Ther 17: 1201-1213, 2006. 